\title{
1 Identification and Quantification of Anthocyanins in Transgenic Purple
}

2 Tomato

3 Xiaoyu Su${ }^{\dagger}$, Jianteng $\mathrm{Xu}^{\dagger}$, Davina Rhodes ${ }^{\dagger}$, Yanting Shen ${ }^{\dagger}$, Weixing Song ${ }^{\ddagger}$, Benjamin Katz ${ }^{£}$,

4 John Tomich ${ }^{£}$, Weiqun Wang*广

5 †Department of Food Nutrition Dietetics \& Health, ${ }^{\star}$ Department of Statistics, ${ }^{£}$ Department of

6 Biochemistry, Kansas State University, Manhattan, KS 66506, USA

7

$8 *$ Corresponding author at: Department of Food Nutrition Dietetics \& Health, Kansas State

9 University, Manhattan, Kansas 66506, USA. Tel.: +1-785-5320153; Fax: +1-785-532-3132; E-

10 mail: wwang@ksu.edu (W. Wang).

11

12 Abbreviations used: Del, Delila; F3'5'H, flavonoid 3'5'-hydroxylas; PAL, phenylalanine

13 ammonia-lyase; Ros1, Rosea1.

14 
15 ABSTRACT: Anthocyanins are natural pigments derived from the phenylpropanoid pathway.

16 Most tomatoes produce little anthocyanins, but the transgenic purple tomato biosynthesizes a

17 high level of anthocyanins due to expression of two transcription factors ( $D e l$ and Ros 1 ). This

18 study was to identify and quantify anthocyanins in this transgenic tomato line. Seven

19 anthocyanins, including two new anthocyanins [malvidin-3-(p-coumaroyl)-rutinoside-5-

20 glucoside and malvidin-3-(feruloyl)-rutinoside-5-glucoside], were identified by LC-MS/MS.

21 Petunidin 3-(trans-coumaroyl)-rutinoside-5-glucoside and delphinidin 3-(trans-coumaroyl)-

22 rutinoside-5-glucoside were the most abundant anthocyanins, making up $86 \%$ of the total

23 anthocyanins. Compared to undetectable anthocyanins in the wild type, the contents of

24 anthocyanins in the whole fruit, peel, and flesh of the Del/Ros 1 -transgenic tomato were 5.2 \pm 0.5 ,

$255.1 \pm 0.5$, and $5.8 \pm 0.3 \mathrm{~g} / \mathrm{kg}$ dry matter, respectively. Anthocyanins were undetectable in the

26 seeds of both wide-type and transgenic tomato lines. Such novel and high levels of anthocyanins

27 obtained in this transgenic tomato may provide unique functional products with potential health

28 benefits.

29 KEYWORDS: Anthocyanins, transgenic tomatoes, Delila; Roseal

\section{1. Introduction}

32 The natural pigments produced in plants, including chlorophylls, carotenoids, and

33 anthocyanins, are generally synthesized via phenylpropanoid and terpenoid pathways (Gonzali et

34 al., 2009). Anthocyanins (derived from Greek anthos (flower) and kyanos (dark blue)) are one of

35 the most important water-soluble plant pigments (Delgado-Vargas \& Paredes-López, 2003).

36 They are synthesized by the flavonoid branch of the phenylpropanoid pathway through

37 secondary metabolism in higher plants. Among over 600 types of anthocyanins (Xu \& Howard, 
2012), the majority of anthocyanin aglycones found in nature consist of six anthocyanidins,

39 i.e.,cyanidin, delphinidin, petunidin, peonidin, pelargonidin, and malvidin. They share a 2-

40 phenylbenzopyrilium (flavyl-ium) skeleton hydroxylated in 3, 5, and 7 positions, with different

41 substitutions at R1 and R2 (Fig. 1). In comparison with other flavonoids, anthocyanins possess a

42 positive charge on its C-ring, which leads to different colors in response to various pH (Wang \&

43 Stoner, 2008).

44 Anthocyanins present in human foods have received considerable attention due to their

45 possible health-promoting properties such as antioxidant and anti-inflammatory effects (Lim et

46 al, 2013; Bowen-Forbes et al, 2010). Based on food intake data from NHANES 2001-2002, the

47 daily intake of anthocyanins was estimated to be $12.5 \mathrm{mg} /$ day/person in the United States (Xu et

48 al., 2006). The predominant dietary anthocyanins are malvidin, delphinidin, and peonidin

49 glycosides (Bognar et al., 2013), which can be found in many plant foods, including berries,

50 purple sweet potatoes, grapes, and wine.

Tomato (Solanumlycopersicum L.) is one of the most important food crops in the world.

52 Its rich red color is due to accumulation of the carotenoid pigments, i.e., lycopene and phytoene,

53 in the peel and flesh (Pannellini et al., 2004; Khachik et al., 2002). However, when compared to

54 anthocyanin-enriched plants, tomatoes generally produce little anthocyanins. Genetic

55 engineering is a powerful approach to induce and enhance biosynthesis of anthocyanins in plants

56 (Schijlen et al., 2004), which has been successfully applied in food crops such as potato and rice

57 (Tanakaand Ohmiya2008; Lukaszewicz et. al., 2004). Several transgenic tomatoes with increased

58 flavonoid levels have also been developed. A transgenic tomato line created by expressing two

59 maize regulatory genes, $L c$ and $C 1$, was reported to produce a high level of flavonols rather than

60 anthocyanins (Bovy et al., 2002). Overexpression of the ANT1gene encoding a MYB 
61 transcription factor was further reported to induce a purple spotting on the epidermis of tomatoes

62 (Mathews et al., 2003). In addition to the ANT1 gene, combining the atv gene with either Aft or

63 Abg was found to generate anthocyanin petunidin-3-(p-coumaryl)-rutinoside-5-glucoside

64 predominantly in the epidermis of tomatoes up to $0.1 \%$ in fresh weight (Peter et al., 2008).

65 Furthermore, expression of two snapdragon (Antirrhinum majus) transcription factors, i.e., Delila

66 ( Del) and Rosea1 (Ros1), in the fruit of transgenic tomatoes activated multiple anthocyanin

67 biosynthesis-related genes, including phenylalanine ammonia-lyase (PAL), and flavonoid 3'5'-

68 hydroxylase $\left(\mathrm{F}^{\prime} 5^{\prime} \mathrm{H}\right)$ (Butelli et al., 2008). The Del/Ros1 transgenic tomato grew normally

69 during the green stage and then started to accumulate purple pigments during the ripening stage,

70 exhibiting an intense and uniform purple color both in the peel and flesh (Butelli et al., 2008).

71 According to the reported methods by Butelli et al. (2008), the Park lab has engineered and

72 produced the Del/Ros 1 transgenic tomato line (Lim et al. 2014). Figure 2 shows the whole, cross-

73 section, and freeze dry of ripe wild-type and transgenic Del/Ros 1 fruits.

74 The objectives of this study were to identify and quantify the anthocyanin profile in this

75 transgenic tomato, and to determine the distribution of anthocyanins in the peel, flesh, and seed

76 of the fruits.

78 2. Materials and methods

79 2.1. Materials

Acetonitrile, methanol $(\mathrm{MeOH})$, and formic acid used in this study at either HPLC grade

81 or analytic grade were purchased from Thermal fisher Scientific (Suwanee, GA). Water used was

82 purified through Barnstead E-Pure Deionization System (Dubuque, IA) and filtered by Millpore 
$830.45 \mu \mathrm{m}$ membrane (Bedford, MA). A standard of Peonidin-3-glucoside chloride was purchased

84 from Sigma-Aldrich (St. Louis, MO).

\section{2.2. Sample preparation and extraction}

Wild type (Solanum lycopersicum L.cv Rubion) and Del/Ros I transgenic purple

87 tomatoes generated in previous studies (Lim et al. 2014) were harvested in the Kansas State

88 University Department of Horticulture greenhouses. For each line, ripe tomatoes were washed

89 with tap water, diced into approximately $0.5 \mathrm{~cm}$ cubes, freeze-dried (Labconco, FreeZone 2.5),

90 and ground by a food processor into powder. Prepared powder was then stored at $-80^{\circ} \mathrm{C}$ until

91 further extraction. For preparation of anthocyanin extracts, $0.05 \mathrm{~g}$ of the powder was extracted

92 with $4 \mathrm{~mL}$ of acidified $\mathrm{MeOH}$ with $1 \mathrm{~N}$ formic acid at 9:1 (v/v). The flasks containing

93 powder/solvent mixture were wrapped with aluminum foil to avoid light exposure. After a 12-

94 hour extraction, the samples were centrifuged at 2,800 rpm for 30min and then the supernatant

95 was collected and dried by vacuum drier at $25^{\circ} \mathrm{C}$ overnight. One $\mathrm{mL}$ of the acidified $\mathrm{MeOH}$ was

96 added and then the dissolved extract was filtered by Whatman syringe filter (Whatman $0.45 \mathrm{um}$

97 PVDF) for LC-MS/MS analysis.

98 2.3. Identification and analysis of anthocyanins by LC-MS/MS

99 LC coupled Electrospray Ionization tandem Mass Spectrometry (LC-MS/MS) was used

100 to carry out anthocyanin identification and quantification. A Shimadzu HPLC system (Kyoto,

101 Japan) was used for chromatographic analysis and separation. This system employed a DGU-

102 20A3 built in degasser, a LC-20AB solvent delivery pump, a SIL-20ACHT auto-sampler, a

103 CTO-20AC column-holding oven, a CBM-20A communicator module, and a SPD-M20A

104 Photodiode Array Detectors. A Waters (Milford, MA) $\mathrm{C}_{18}$ reversed phase column (250 mm

105 length, $4.6 \mathrm{~mm}$ diameter) was used for anthocyanin separation. Data was analyzed using LC 
106 solution software (Kyoto, Japan). Elution was performed with mobile phase A (5\%formic acid in

107 de-ionized water) and mobile phase B (5\% formic acid in acetonitrile/water 1:1 v: v). An

108 optimum column temperature was set at $25^{\circ} \mathrm{C}$. At a flow rate of $0.8 \mathrm{~mL} / \mathrm{min}$, the gradient

109 conditions were set as follows: solvent B volume at 5-20\% for 35min, 20-50\% for 10min, and

110 held at $50 \%$ for $10 \mathrm{~min}$ before returning to $5 \%$ at $60 \mathrm{~min}$. The detector performed a full spectrum

111 scan between 190-800nm, where $520 \mathrm{~nm}$ was used for monitoring anthocyanins. Peonidin-3-

112 glucoside was used as an internal standard for quantitation of extraction recovery and the

113 anthocyanin contents were expressed as peonidin 3-glucoside equivalent (PN3GE). Based on a

114 signal-to-noise ratio of 3:1 and the standard deviation of the lowest concentration of PN3G/slope

115 of the calibration line, the detection limit was estimated to be 2 pmol.

116 Mass spectrometric scan was performed on a Bruker Esquire 3000 in positive mode with

117 a scanning interval $500-1200 \mathrm{~m} / \mathrm{z}$. Nebulization was conducted at $350{ }^{\circ} \mathrm{C}$ aided by concurrent $\mathrm{N}_{2}$

118 flow at 10 psi; capillary and cone voltages were set at $3.5 \mathrm{kV}$ and $40 \mathrm{~V}$; drying gas flow rate was

$1195 \mathrm{~L} / \mathrm{min}$. Mass of precursor ions and reactions of fragments loss were evaluated. Data were

120 analyzed using Bruker Hystar Post Processing software (Bruker Daltonics, GmbH, Billerica,

121 MA). The ESI/MS data was used to confirm the mass of each anthocyanin HPLC peak. The mass

122 spectrometry instrument was controlled by the esquire control 5.3 software (Bruker Daltonics,

$123 \mathrm{GmbH}$, Billerica, MA) and the data were processed with Data analysis 3.3 software (Bruker

124 Daltonics, GmbH, Billerica, MA). Individual identification of each anthocyanin was

125 accomplished by comparison of HPLC retention time, absorbance spectra, and MS spectra with

126 our previously published anthocyanin data (Lim et al. 2013; Xu et al. 2015). The new

127 anthocyanins were identified by matching the mass spectral data with those from the National 
128 Institute of Standards and Technology Mass Spectra Library data (NIST08, National Institute of

129 Standards and Technology, Gaithersburg, MD, USA).

$130 \quad$ 2.4. Statistical analysis

131 Data were analyzed using SAS statistical software, version 9.3 (SAS Institute, Cary, NC,

132 USA). Results were evaluated by one-way ANOVA using a general linear model procedure

133 followed by Tukey's post-hoc test. The results were presented as means \pm SD, and a probability

134 at $p \leq 0.05$ was considered significant.

\section{Results and discussion}

\subsection{Chromatographic separation}

The objectives of this study were focused on characterizing the anthocyanin profile in a transgenic purple tomato and quantifying the anthocyanin content in each part of the tomato

140 using HPLC-MS/MS. The profile of the anthocyanin peaks from transgenic tomato Del/Ros 1

141 was shown by HPLC chromatogram in Fig. 3. While no anthocyanin peaks were detectable in

142 the wild type, a total of seven peaks were eluted at the retention times between 23 and 38 min in

143 the transgenic Del/Ros 1 fruits. Of these, peaks 2 and 4 were the major anthocyanins and their

144 peak areas appeared to be more than half of the total anthocyanin peak areas.

\section{3.2. Mass spectrometric identification}

146 Following HPLC separation, LC-MS/MS data were characterized by monitoring the

147 molecular ion characteristics for each peak. The $\mathrm{m} / \mathrm{z}$ ratio of each intact anthocyanin and its

148 daughter fragments are listed in Table 1. As shown in Table 1, delphinidin (Dpd m/z 302),

149 petunidin $(\mathrm{Ptd} \mathrm{m} / \mathrm{z} 316)$, and malvidin $(\mathrm{Mv} \mathrm{m} / \mathrm{z} 331)$ were the three anthocyanidin aglycones

150 detected in the transgenic tomato line. Five of the seven anthocyanins including delphinidins 
151 (peaks 1-3) and petunidins (peaks 4-5) were reported previously by Bultelli et al. (2008).

152 However, two new malvidins (peaks 6-7) were found in the transgenic tomato line for the first 153 time. As shown in Figure 4a, the ions of peak 6, i.e., malvidin-3-(p-coumaroyl)-rutinoside-5154 glucoside (m/z 947), produced three fragments of $\mathrm{m} / \mathrm{z} 785,493$, and 331. Transition 947 to 785 155 and 947 to 493 implied the loss of glucose (m/z 162) and p-coumaroyl (m/z 454), respectively. 156 Transition 947 to 331 produced malvidin aglycone (m/z 331) caused by the loss of glucose and 157 p-coumaroyl. In Figure 4b, malvidin-3-(feruloyl)-rutinoside-5-glucoside (peak 7) produced 158 transitions of 977 to 815,493 , and $331 \mathrm{~m} / \mathrm{z}$. Transition 977 to 815 and 947 to 493 indicated the 159 loss of glucose (m/z 162) and feruloyl (m/z 484), respectively, while transition 947 to 331 160 produced malvidin aglycone $(\mathrm{m} / \mathrm{z} 331)$.

161 When compared with other glycosylated anthocyanidins, malvidins have been found to 162 have stronger inhibitory effects on nitric oxide production in LPS/IFN- $\gamma$-activated RAW 264.7 163 mouse macrophage cells due to better absorption and better free radical scavenging activity 164 (Wang and Mazza, 2002). In addition, possible health benefits of dietary malvidins have been 165 reported because of anti-proliferative (Seeram \& Zhang, 2003; Hyun \& Chung, 2004) and anti166 inflammatory activities (Jing et al. 2008; Wedick et al. 2012). The total content of anthocyanins 167 in the Del/Ros 1 transgenic tomato is equally distributed, with $5.1 \pm 0.5 \mathrm{~g} / \mathrm{kg} \mathrm{DW}$ in the peel and $1685.8 \pm 0.3 \mathrm{~g} / \mathrm{kg}$ DW in the flesh. Total anthocyanin contents in Del/Rosl are near $5.2 \pm 0.5 \mathrm{~g}$ $169 \mathrm{PN} 3 \mathrm{GE} / \mathrm{kg} \mathrm{DW}$, or $0.5 \%$ of dry weight, which is higher than some of the anthocyanin-enriched 170 foods such as red raspberry (3.9 g/kg DW by Wang \& Lin 2000), strawberry (3.2 g/kg DW by 171 Wang \& Lin 2000), and mulberry (2.1 g/kg DW by Bae \& Suh, 2007).

172 The reason why two new anthocyanins could be detected in the transgenic line may relate 173 to the modified extraction and HPLC method that allowed for a distinct peak separation. In this 
174 study, $10 \%$ of formic acid was added to the methanol before extraction, creating a low $\mathrm{pH}$

175 environment for anthocyanin stabilization. The decrease of flow rate and solvent B ratio during

176 the gradient elution might also provide better peak separation. Lastly, with a C18 stationary

177 phase column, more polar solvent A and less polar solvent B mobile phase may carry out a better 178 gradient elution.

\section{3.3. Anthocyanin quantification in transgenic tomato}

While anthocyanins were undetectable in the wild-type, the contents of anthocyanins in

181 the whole fruit, peel, and flesh of the Del/Rosl-transgenic tomato were 5.2 $\pm 0.5,5.1 \pm 0.5$, and

$1825.8 \pm 0.3 \mathrm{~g} / \mathrm{kg}$ dry matter, respectively. Anthocyanins were undetectable in the seeds of both

183 wide-type and transgenic tomato lines.

Table 2 lists the content profile of individual anthocyanin in the whole, peel, and flesh of

185 the transgenic tomato line. The predominant anthocyanins were delphinidin-3-(trans-coumaroyl)-

186 rutinoside-5-glucoside and petunidin-3-(trans-coumaroyl)-rutinoside-5-glucoside, which

187 contributed to nearly $86 \%$ of the total anthocyanins. The reason why they are the highest among

188 all the anthocyanins is not clear, but it may be due to the transgenic Del/Ros 1 -induced

189 overexpression of the genes that relate to the specific anthocyanin biosynthesis pathway in which

190 delphinidin-3-(trans-coumaroyl)-rutinoside-5-glucoside is an immediate precursor for petunidin-

191 3-(trans-coumaroyl)-rutinoside-5-glucoside (Holton \& Cornish 1995). Two new anthocyanins,

192 malvidin-3-(p-coumaroyl)-rutinoside-5-glucosideand malvidin-3-(feruloyl)-rutinoside-5-

193 glucoside made up $6 \%$ of the total anthocyanins.

194 In conclusion, seven anthocyanins, including 2 new anthocyanins, have been identified in

195 the Del/Ros1 transgenic tomato. Compared to undetectable anthocyanins in the wild type, the

196 Del/Ros1 transgenic tomato produced a high level of anthocyanins that may provide unique 
197 functional products with potential health benefits.

198

199 Acknowledgments

The authors greatly thank Dr. Sunghun Park (Kansas State University Department of

201 Horticulture) for providing wild type and transgenic tomato samples for anthocyanin analysis.

202 This study was supported in part by a USDA Cooperative Project KS511-1001903 from the

203 Agricultural Experiment Station, Kansas State University (Contribution \#15-329-J from the

204 Kansas Agricultural Experiment Station).

205 


\section{References}

207 Bognar, E., Sarszegi, Z.,et al. (2013). Antioxidant and Anti-Inflammatory Effects in RAW264.7 Macrophages of Malvidin, a Major Red Wine Polyphenol. PLOS ONE, 8(6), 1.

Bae, S. H., Suh, H. J. (2007). Antioxidant activities of five different mulberry cultivars in Korea. LWT - Food Science and Technology, 40(6), 955-962.

Delgado-Vargas, F. O., P-L. (2003). Anthocyaninsand betalains. In Natural Colorants for Food and Nutraceutical Uses, Ch.8, 167-219

Etminan, M., Takkouche, B., Caamaño-Isorna, F. (2004). The role of tomato products and lycopene in the prevention of prostate cancer: a meta-analysis of observational studies. Cancer Epidemiology, Biomarkers \& Prevention, 13(3), 340-345.

Gonzali1, S., Mazzucato, A., Perata1, P. (2009). Purple as a tomato: towards high anthocyanin tomatoes. TRENDS in Plant Science, 14(5), 237-241.

Holton, T.A., Cornish, E.C. (1995). Genetics and biochemistry of anthocyanin biosynthesis. Plant Cell, 7, 1071-1083. 
Hyun, J.W., Chung, H. S. (2004). Cyanidin and Malvidin from Oryza sativa cv. Heugjinjubyeo mediate cytotoxicity against human monocytic leukemia cells by arrest of G (2)/M phase and induction of apoptosis. Journal of agricultural and food chemistry, 52(8), 2213-2217.

232 Jing, P., Bomser, J.A., Schwartz, S.J., He, J., Magnuson, B.A., Giusti, M.M. (2008). Structureinhibition of colon cancer cell growth. Journal of Agricultural and Food Chemistry, 56(20), 9391-9398.

Khachik, F., Carvalho, L., Bernstein, P.S., Muir, G.J., Zhao, D.Y., Katz, N.B. (2002). Chemistry, distribution, and metabolism of tomato carotenoids and their impact on human health. Experimental Biology and Medicine, 227(10), 845-851.

Lim, S., Xu, J., Kim J, Chen, T. et al. (2013). Role of anthocyanin-enriched purple-fleshed sweet potato p40 in colorectal cancer prevention. Molecular Nutrition \& Food Research, 57(11), 1908-1917.

Lim, W., Miller, R., Park, J., Park, S. (2014). Consumer sensory analysis of high flavonoid transgenic tomatoes. Journal of Food Science, 79:S1212-1217.

Lukaszewicz, M., Matysiak-Kata, I., Skala, J., Fecka, I., Cisowski, W., Szopa, J. (2004). Antioxidant capacity manipulation in transgenic potato tuber by changes in phenolic compounds content. Journal of Agricultural and Food Chemistry, 52(6), 1526-1533.

Mathews, H., Clendennen, S. K., Caldwell, C. G. et al. (2003). Activation Tagging in Tomato Identifies a Transcriptional Regulator of Anthocyanin Biosynthesis, Modification, and Transport. Plant Cell. 15(8), 1689-1703. 
Mes, P. J., Boche, P., Myers, J. R. (2008). Characterization of Tomatoes Expressing

Anthocyanin in the Fruit. Journal of the American Society for Horticultural Science, 133(2), 262-269.

253

254

255

256

257

258

259

260

261

262

263

264

265

266

267

268

269

270

271

272

Pannellini, T., Iezzi, M., Liberatore, M. et al. (2010). A dietary tomato supplement prevents prostate cancer in TRAMP mice. Cancer Prevention Research, 3(10), 1284-1291.

Schijlen, E. G., Ric de Vos, C. H., van Tunen, A. J., Bovy A. G. (2004). Modification of flavonoid biosynthesis in crop plants. Phytochemistry, 65(19), 2631-2648.

Seeram, N. P.,Zhang,Y.,Nair, M. G. (2003). Inhibition of proliferation of human cancer cells and cyclooxygenase enzymes by anthocyanidins and catechins. Nutrition and Cancer, 46(1), 101-106.

Tanaka, Y., Ohmiya, A. (2008). Seeing is believing: engineering anthocyanin and carotenoid biosynthetic pathways. Current opinion in Biotechnology, 19(2), 190-197.

Wang, J., Mazza, G. (2002). Inhibitory effects of anthocyanins and other phenolic compounds on nitric oxide production in LPS/IFN-gamma-activated RAW 264.7 macrophages. Journal of Agricultural and Food Chemistry, 50(4), 850-857.

Wang, L. S., Stoner, G. D. (2008). Anthocyanins and their role in cancer prevention. Cancer Letters, 269(2), 281-290.

Wedick, M.N., Pan, A., Cassidy, A., Rimm, E.B. (2012). Dietary flavonoid intakes and risk of type 2 diabetes in US men and women. The American Journal of Clinical Nutrition, 95(4), 925-933.

Wu, X., Beecher, G. R., Holden, J. M., Haytowitz, D. B., Gebhardt, S. E., Prior, R. L. (2006). Concentrations of anthocyanins in common foods in the United States and estimation of normal consumption. Journal of Agricultural and Food Chemistry, 54(11), 4069-4075. 
273 Xu J, Su X, Lim S, Griffin J, Carey E, Katz B, Tomich J, Smith JS, Wang W. (2015).

274 Characterization and stability of anthocyanins in purple-fleshed sweet potato P40. Food $275 \quad$ Chemistry, 186(11), 90-96.

276 Xu, Z. Howard, L. (2012). Analysis Methods of Anthocyanins. Analysis of Antioxidant-Rich

$277 \quad$ Phytochemicals, Ch. 5, 149-180.

278 


\section{Figure Legends}

280 Figure 1. Structures of common anthocyanidins and anthocyanins

281 Figure 2. Representative images of the whole, cross-section, and freeze dry of the ripe wild-type 282 (left column) vs. the transgenic Del/Ros 1 tomato fruit (right column).

283 Figure 3. Representative HPLC chromatograms of anthocyanins in the wild-type and the

284 transgenic Del/Ros 1 tomatoes (the peak number corresponding to anthocyanin name is shown in 285 Table 1).

286 Figure 4. Mass spectrometric data of two new malvidins detected in the transgenic Del/Ros 1 287 purple tomato: a) Malvidin-3-(p-coumaroyl)-rutinoside-5-glucoside; b) malvidin-3- (feruloyl) 288 rutinoside-5-glucoside (Mv, Malvidin; Glc, glucose; 3FR, 3-(feruloyl)-rutinoside; 3PR, 3-(p289 coumaroyl)-rutinoside). 
Table 1. Mass Spectrometric Data of Anthocyanins in the Transgenic Del/Ros1 Tomato

\begin{tabular}{|c|c|l|c|l|}
\hline $\begin{array}{c}\text { Peak } \\
\#\end{array}$ & $\begin{array}{c}\text { Retention time } \\
(\mathrm{min})\end{array}$ & \multicolumn{1}{|c|}{ Anthocyanins } & $\begin{array}{c}\text { [M+H }]^{+} \\
(\mathrm{m} / \mathrm{z})\end{array}$ & \multicolumn{1}{|c|}{ Detected fragments* $^{*}$} \\
\hline 1 & 24.67 & $\begin{array}{l}\text { Delphinidin-3-(caffeoyl)- } \\
\text { rutinoside-5-glucoside }\end{array}$ & 935 & $\begin{array}{l}773(\mathrm{Dpd}+3 \mathrm{CR}), 465 \\
(\mathrm{Dpd}+\mathrm{Glc}), 302(\mathrm{Dpd})\end{array}$ \\
\hline 2 & 28.17 & $\begin{array}{l}\text { Delphinidin-3-(trans- } \\
\text { coumaroyl)-rutinoside-5- } \\
\text { glucoside }\end{array}$ & 919 & $\begin{array}{l}757(\mathrm{Dpd}+3 \mathrm{TR}), 465 \\
(\mathrm{Dpd}+\mathrm{Glc}), 302(\mathrm{Dpd})\end{array}$ \\
\hline 3 & 29.80 & $\begin{array}{l}\text { Delphinidin-3-(feruloyl)- } \\
\text { rutinoside-5-glucoside }\end{array}$ & 949 & $\begin{array}{l}787(\mathrm{Dpd}+3 \mathrm{FR}), 464 \\
(\mathrm{Dpd}+\mathrm{Glc}), 303(\mathrm{Dpd})\end{array}$ \\
\hline 4 & 31.77 & $\begin{array}{l}\text { Petunidin-3-(trans-coumaroyl)- } \\
\text { rutinoside-5-glucoside }\end{array}$ & 933 & $\begin{array}{l}772(\mathrm{Ptd}+3 \mathrm{TR}), 479 \\
(\mathrm{Ptd}+\mathrm{Glc}), 316(\mathrm{Ptd})\end{array}$ \\
\hline 5 & 33.26 & $\begin{array}{l}\text { Petunidin-3-(feruloyl)- } \\
\text { rutinoside-5-glucoside }\end{array}$ & 963 & $\begin{array}{l}800(\mathrm{Ptd}+3 \mathrm{FR}), 478 \\
(\mathrm{Ptd}+\mathrm{Glc}), 316(\mathrm{Ptd})\end{array}$ \\
\hline 6 & 34.39 & $\begin{array}{l}\text { Malvidin-3-(p-coumaroyl)- } \\
\text { rutinoside-5-glucoside }\end{array}$ & 947 & $\begin{array}{l}785(\mathrm{Mv}+3 \mathrm{PR}), 493(\mathrm{Mv} \\
+\mathrm{Glc}), 331(\mathrm{Mv})\end{array}$ \\
\hline 7 & 36.81 & $\begin{array}{l}\text { Malvidin-3-(feruloyl)- } \\
\text { rutinoside-5-glucoside }\end{array}$ & 977 & $\begin{array}{l}815(\mathrm{Mv}+3 \mathrm{FR}), 493(\mathrm{Mv} \\
+\mathrm{Glc}), 331(\mathrm{Mv})\end{array}$ \\
\hline
\end{tabular}

*Dpd, delphinidin; Ptd, petunidin; Mv, malvidin; Glc, glucose; 3CR, 3-(caffeoyl)-rutinoside;

3TR, 3-(trans-coumaroyl)-rutinoside; 3FR, 3-(feruloyl)-rutinoside; 3PR, 3-(p-coumaroyl)-

rutinoside. 
Table 2. The contents of individual anthocyanin ( $\mathrm{mg} / \mathrm{kg}$ dry matter) in the whole fruit, flesh and peel of the transgenic Del/Rosl tomato

\begin{tabular}{lccc}
\hline & Whole & Peel & Flesh \\
\hline $\begin{array}{l}\text { Delphinidin3-(caffeoyl)- } \\
\text { rutinoside-5-glucoside }\end{array}$ & $136.7 \pm 10.9^{\mathrm{a}}$ & $84.9 \pm 7.0^{\mathrm{b}}$ & $153.6 \pm 11.5^{\mathrm{c}}$ \\
$\begin{array}{l}\text { Delphinidin3-(trans- } \\
\text { coumaroyl)-rutinoside-5- } \\
\text { glucoside }\end{array}$ & $1434.6 \pm 144.2^{\mathrm{a}}$ & $1145.3 \pm 115.0^{\mathrm{b}}$ & $1624.3 \pm 104.5^{\mathrm{a}}$ \\
$\begin{array}{l}\text { Delphinidin3-(feruloyl)- } \\
\text { rutinoside-5-glucoside }\end{array}$ & $53.4 \pm 6.3^{\mathrm{a}}$ & $25.5 \pm 2.8^{\mathrm{b}}$ & $64.9 \pm 4.0^{\mathrm{c}}$ \\
$\begin{array}{l}\text { Petunidin3-(trans- } \\
\text { coumaroyl)-rutinoside-5- } \\
\text { glucoside }\end{array}$ & $2849.6 \pm 303.7^{\mathrm{a}}$ & $3325.1 \pm 351.4^{\mathrm{a}}$ & $3021.6 \pm 156.5^{\mathrm{a}}$ \\
$\begin{array}{l}\text { Petunidin 3-(feruloyl)- } \\
\text { rutinoside-5-glucoside } \\
\text { malvidin-3-(p- }\end{array}$ & $446.6 \pm 75.1^{\mathrm{a}}$ & $233.8 \pm 11.9^{\mathrm{b}}$ & $554.2 \pm 30.2^{\mathrm{c}}$ \\
$\begin{array}{l}\text { coumaroyl)- } \\
\text { rutinoside-5-glucoside } \\
\text { malvidin-3-(feruloyl) - } \\
\text { rutinoside-5-glucoside }\end{array}$ & $240.0 \pm 15.4^{\mathrm{a}}$ & $270.6 \pm 23.0^{\mathrm{a}}$ & $259.0 \pm 27.7^{\mathrm{a}}$ \\
\hline
\end{tabular}

*Data are expressed as mean \pm SD $(n=4-6)$. Values marked by different letters within same row indicate significant difference $(p<0.05)$. 


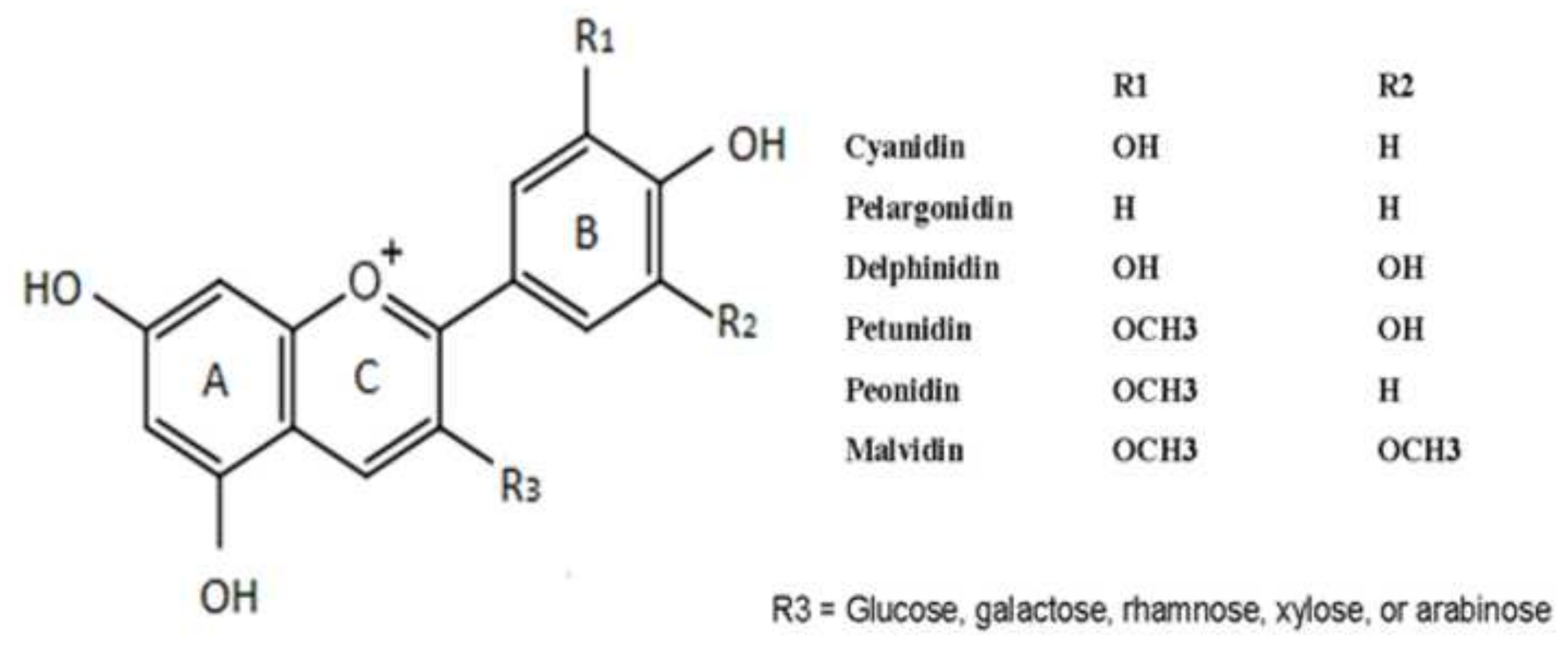


Wild
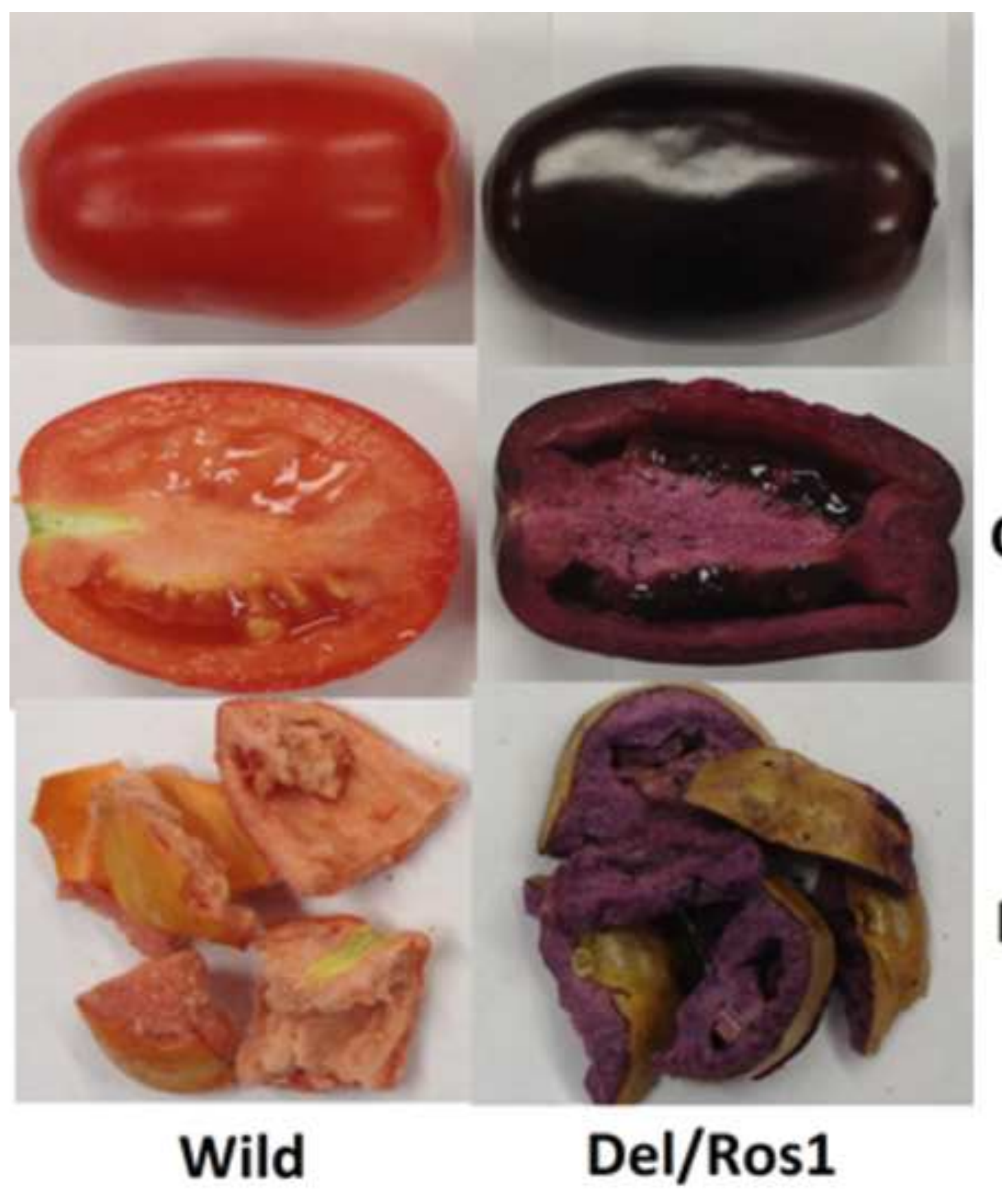

Del/Ros1

\author{
Whole \\ Freeze Dry

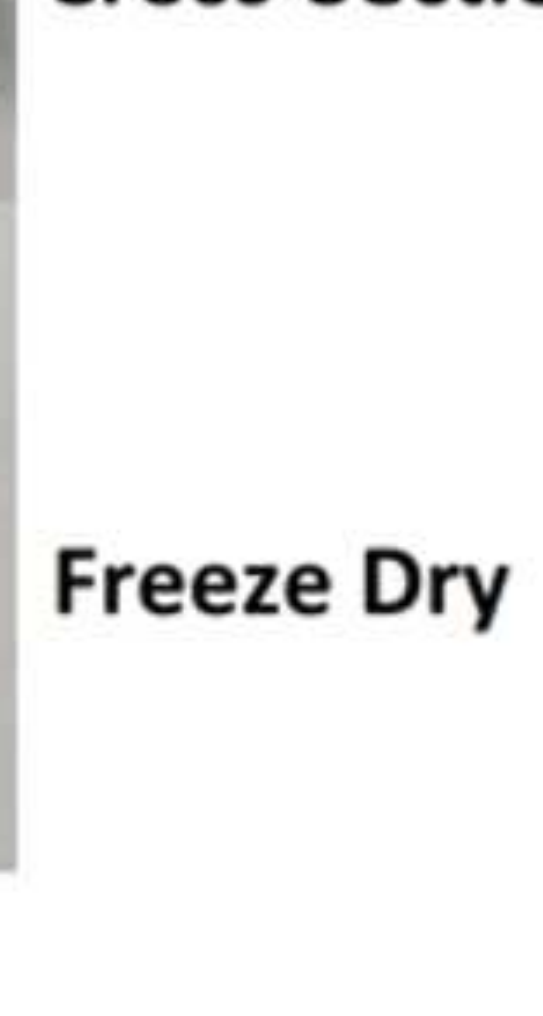 \\ Whole

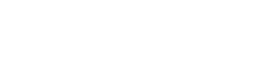

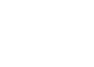

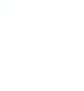

.

.




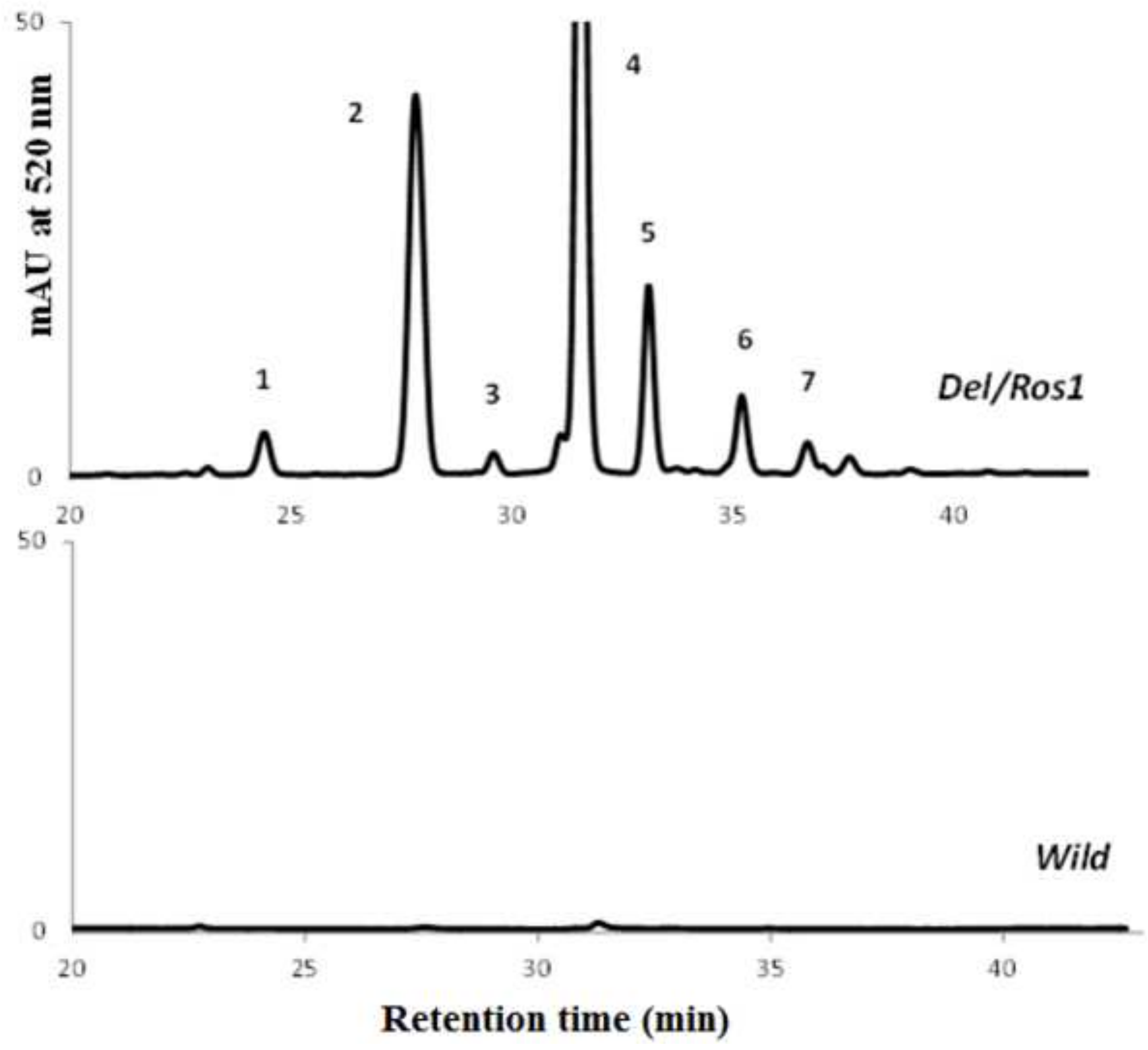


